\title{
Particle Production Studies at LHCb
}

\author{
Christopher Blanks*, on behalf of the LHCb collaboration \\ Imperial College, London, United Kingdom \\ E-mail: c.blanks07@imperial.ac.uk
}

\begin{abstract}
The LHCb detector is introduced as a precision tool for tracking and particle identification in the forward pseudorapidity region, $2<\eta<5$. Preliminary measurements are presented from $p p$ collisions delivered by the LHC for the production cross section of $K_{S}^{0}$ at $\sqrt{s}=0.9 \mathrm{TeV}$ and the production ratios $\bar{\Lambda} / \Lambda, \bar{\Lambda} / K_{S}^{0}$ and $\bar{p} / p$ at both 0.9 and $7 \mathrm{TeV}$. These preliminary results are given as a function of transverse momentum, $p_{T}$, and rapidity, $y$ (or pseudorapidity, $\eta$ ) and are compared with tunings of the PYTHIA event generator. There is broad agreement at $7 \mathrm{TeV}$ however an excess in strange particle production is observed at $0.9 \mathrm{TeV}$. This excess suggests an underestimate in simulation of either the strange quark production fraction or the total particle multiplicity. The baryon ratio results are also presented in terms of the rapidity loss, $\Delta y$, and compatibility with previous measurements is observed.
\end{abstract}

35th International Conference of High Energy Physics

July 22-28, 2010

Paris, France

* Speaker. 


\section{Introduction}

The Large Hadron Collider Beauty experiment (LHCb) at CERN is a single-arm spectrometer with full instrumentation of the high pseudorapidity region $2<\eta<5$. Vertexing near the interaction region is made to a precision of $\sim 50(150) \mu \mathrm{m}$ by the Vertex Locator (VELO) for primary (secondary) vertices. The tracking design provides $97 \%$ reconstruction efficiency and a momentum resolution $\delta p / p \sim 0.4 \%$ for "Long tracks" which pass from the VELO through the $4 \mathrm{Tm}$ warm dipole magnet to the downstream tracking stations. Particle identification (PID) is provided by a Ring Imaging Cherenkov (RICH) system over the momentum range 2 to $\sim 100 \mathrm{GeV} / c$.

LHCb recorded a data set of $6.8 \pm 0.1 \mu \mathrm{b}^{-1}$ from the LHC's 2009 pilot run at $\sqrt{s}=0.9 \mathrm{TeV}$ and, by Summer 2010, had accumulated integrated luminosities of $0.3 \mathrm{nb}^{-1}$ and $0.2 \mathrm{nb}^{-1} p p$ collisions at $\sqrt{s}=0.9 \mathrm{TeV}$ and $7 \mathrm{TeV}$ respectively. These data sets were recorded with a loose trigger requiring either a minimum energy deposition in the calorimeters or the fast reconstruction of at least one track and represent a valuable opportunity to study minimum bias physics.

While the underlying physical interactions of particle collisions are known, they cannot be calculated reliably. Phenomenological models, or "generators", have therefore been developed and are tuned to replicate experimental observations. Measurements at LHCb will probe new energy regimes for $p p$ collisions and will be of particular value due to a unique coverage at high $\eta(y)$.

Four measurements are presented in this paper: the production cross section of $K_{S}^{0}$ at $\sqrt{s}=$ $0.9 \mathrm{TeV}$ and the production ratios $\bar{\Lambda} / \Lambda, \bar{\Lambda} / K_{S}^{0}$ and $\bar{p} / p$ at $\sqrt{s}=0.9 \mathrm{TeV}$ and $7 \mathrm{TeV}$. Preliminary results are compared with tunings of the PYTHIA [1] generator.

\section{2. $K_{S}^{0}$ production cross section}

Strangeness production offers a promising testing ground for phenomenological collision models since strange quarks must be created in hadronisation. $K_{S}^{0}$ production in particular offers an ideal early measurement for $\mathrm{LHCb}$ with high-purity selections possible from minimum bias data using only the tracking system. The full analysis procedure and results are detailed elsewhere [2]. $K_{S}^{0}$ are selected from the 2009 pilot run data set using the decay $K_{S}^{0} \rightarrow \pi^{+} \pi^{-}$. The two halves of the VELO were retracted from the interaction point to accommodate the significant beam broadening at low energy, resulting in a reduced acceptance. A selection was therefore developed without VELO hits, using all reconstructed tracks to complement the high precision Long track results. The results are presented in figure 2 and show good agreement with model predictions. The largest systematic errors come from the measurement of the integrated luminosity $(\sim 12 \%)$ and the tracking efficiency $(\sim 10 \%)$. The uncertainty on the luminosity is dominated by the limited knowledge of the beam currents in this low intensity pilot run [3]. The uncertainty on the tracking efficiency is determined from the data and is limited by the low statistics of the samples available [4].

\section{3. $V^{0}$ production ratios}

As well as providing the rate of strange particle production at the LHC, LHCb can measure the associated baryon $v s$. meson suppression factor through the production ratio $\bar{\Lambda} / K_{S}^{0}$. Also, the transport of baryon number from colliding protons to final state hadrons can be measured through 


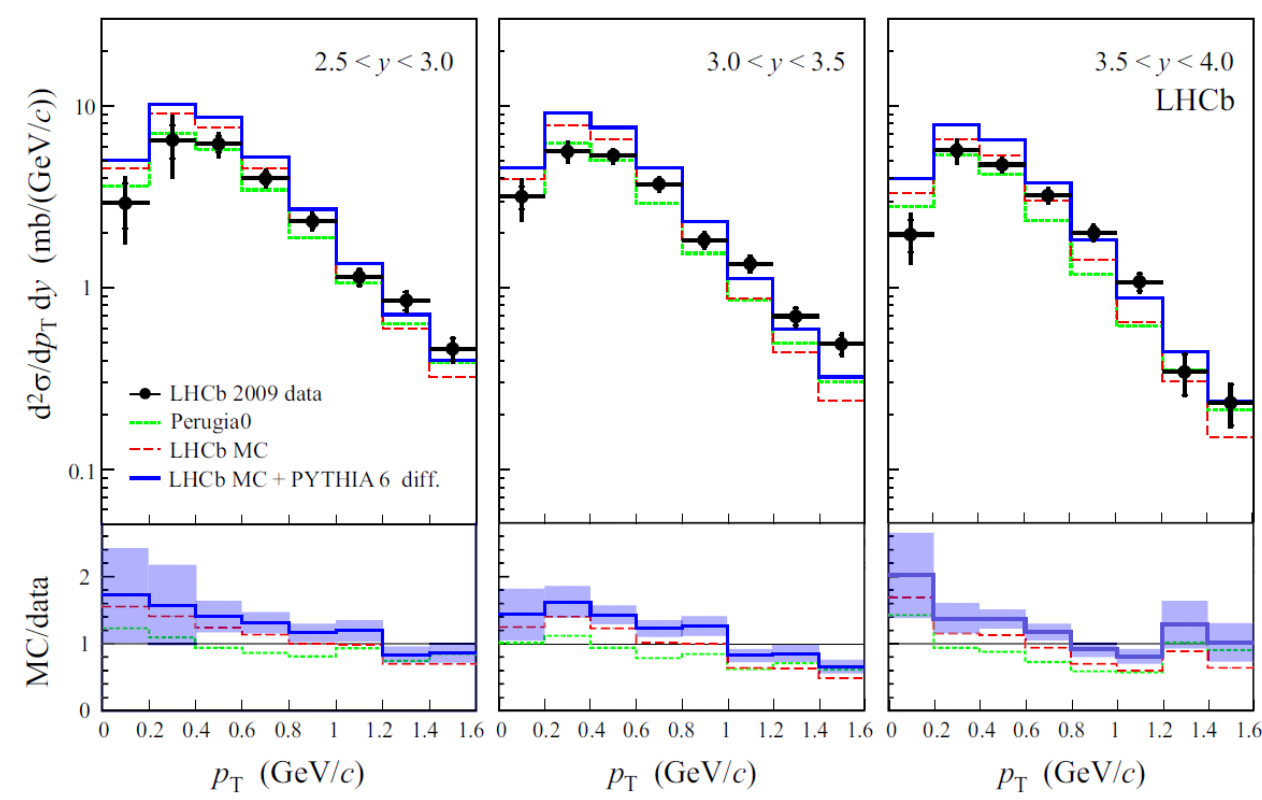

Figure 1: The prompt $K_{S}^{0}$ production cross section from $6.8 \pm 0.1 \mu \mathrm{b}^{-1}$ of $p p$ collisions at $\sqrt{s}=0.9 \mathrm{TeV}$ as a function of $p_{T}$ in three bins of rapidity, $y$. The thick and thin vertical error bars show, respectively, the statistical and total uncertainties. The results from data are compared to the standard candle PYTHIA tuning Perugia 0 [5] and LHCb's own MC, with and without diffractive events.

the ratio $\bar{\Lambda} / \Lambda$. The full analysis procedure and results are detailed in a report to this conference [6]. $V^{0}$ are selected by their dominant decays: $\Lambda \rightarrow p \pi^{-}, \bar{\Lambda} \rightarrow \bar{p} \pi^{+}$and $K_{S}^{0} \rightarrow \pi^{+} \pi^{-}$. A high purity selection of prompt $V^{0}$ is achieved using a Fisher discriminant of impact parameters with respect to the primary vertex. To remove the background of $K_{S}^{0}$ mis-reconstructed as $\Lambda$ or $\bar{\Lambda}$, a $K_{S}^{0}$ mass veto is applied on the invariant mass recalculated using a $\pi \pi$ daughter hypothesis. Preliminary results are presented in figure 3. Systematic uncertainties are estimated at $\sim 2 \%$ for the ratio $\bar{\Lambda} / \Lambda$ and $2-12 \%$ for $\bar{\Lambda} / K_{S}^{0}$. Both ratio measurements benefit with respect to the $K_{S}^{0}$ cross section with no need for the absolute integrated luminosity and the most important remaining systematic is due to the interaction cross sections of the $V^{0}$ and daughters with the detector bulk, which is estimated to be $\mathscr{O}(10 \%)$. Baryon number transport as measured by $\bar{\Lambda} / \Lambda$ is consistent with predictions at $\sqrt{s}=7 \mathrm{TeV}$ but is significantly underestimated at $0.9 \mathrm{TeV}$. Baryon suppression as measured by $\bar{\Lambda} / K_{S}^{0}$ is significantly overestimated at both energies.

\section{4. $\bar{p} / p$ production ratio}

Baryon number transport can also be measured in the production ratio $\bar{p} / p$. The full analysis procedure and results are detailed in a report to this conference [7]. High purity proton selection requires RICH PID, which is calibrated with samples of $\pi, p$ and $K$ from $K_{S}^{0}, \Lambda$ and $\phi$ decays [8]. Preliminary results are presented in figure 4 . The dominant systematic uncertainty arises from the PID, which is most significant at high $\eta$ where the available calibration samples are smaller and is estimated to be $3-14 \%$. Baryon suppression as measured by $\bar{p} / p$ is in good agreement with MC predictions at $\sqrt{s}=7 \mathrm{TeV}$ but an overestimation is observed at $0.9 \mathrm{TeV}$, particularly at lower $p_{T}$. 


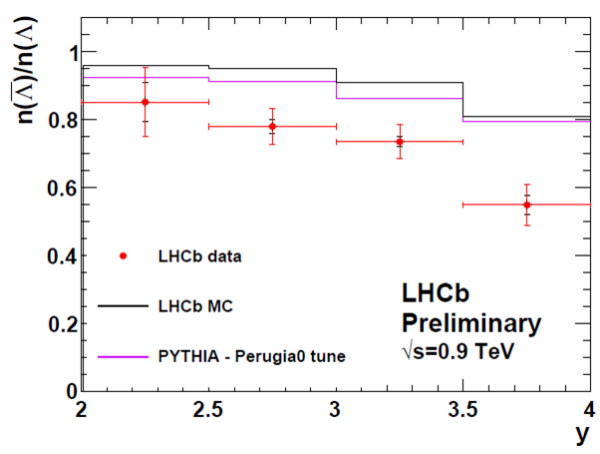

(a) $\bar{\Lambda} / \Lambda$ at $\sqrt{s}=0.9 \mathrm{TeV}$

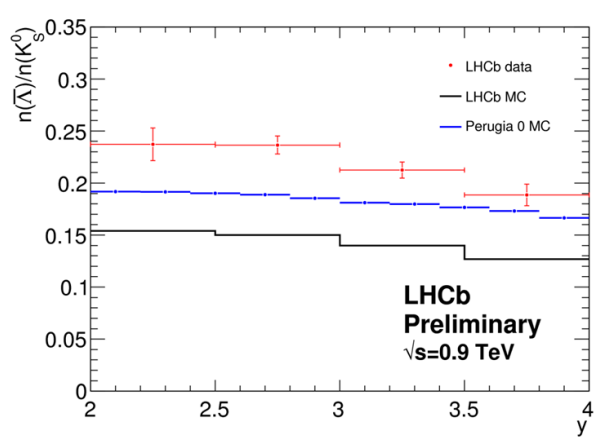

(c) $\bar{\Lambda} / K_{S}^{0}$ at $\sqrt{s}=0.9 \mathrm{TeV}$

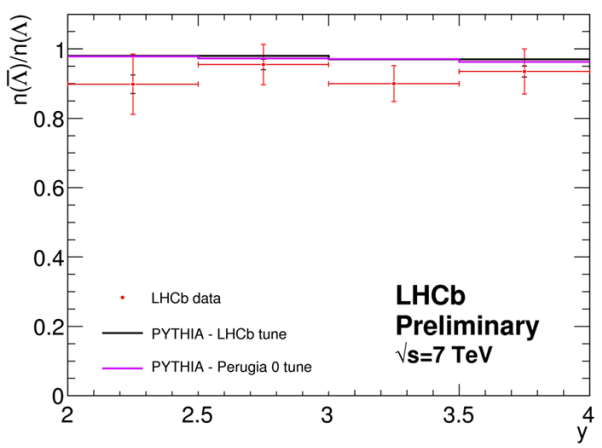

(b) $\bar{\Lambda} / \Lambda$ at $\sqrt{s}=7 \mathrm{TeV}$

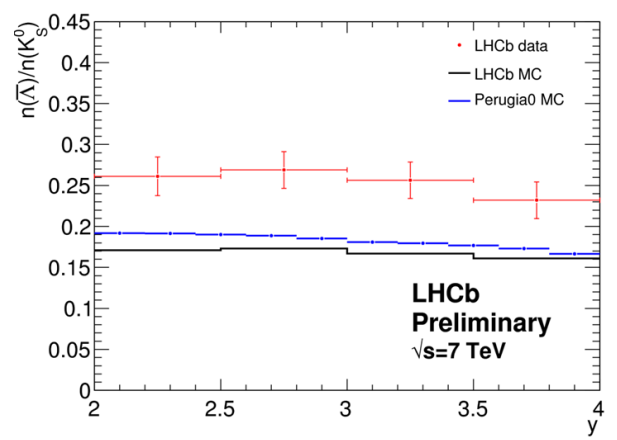

(d) $\bar{\Lambda} / K_{S}^{0}$ at $\sqrt{s}=7 \mathrm{TeV}$

Figure 2: The production ratios $\bar{\Lambda} / \Lambda$ and $\bar{\Lambda} / K_{S}^{0}$ in $p p$ collisions at $\sqrt{s}=0.9 \mathrm{TeV}$ and $7 \mathrm{TeV}$ are compared to the predictions of PYTHIA tunings Perugia 0 and LHCb MC as a function of rapidity, $y$. The vertical error bars give the total statistical and systematic uncertainties.

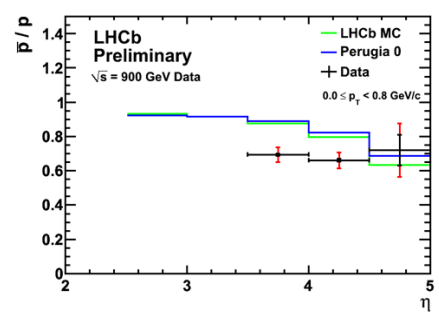

(a)

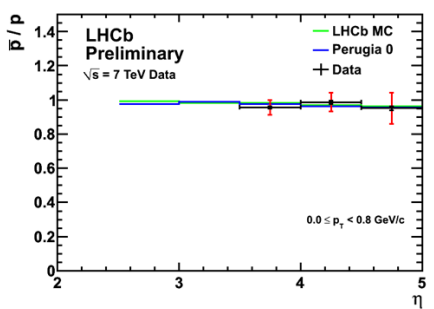

(d)

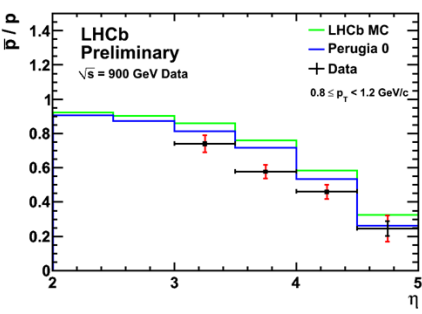

(b)

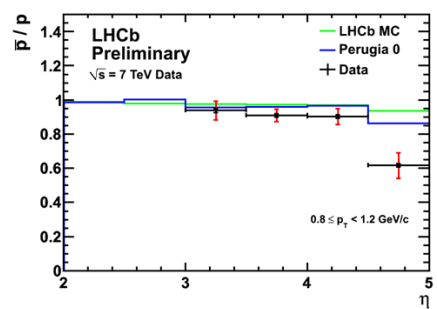

(e)

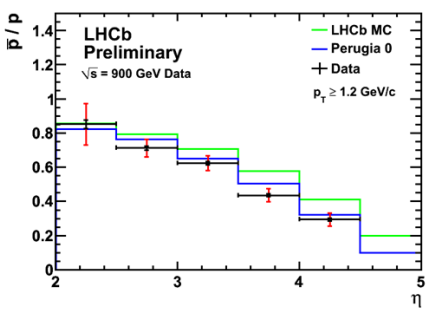

(c)

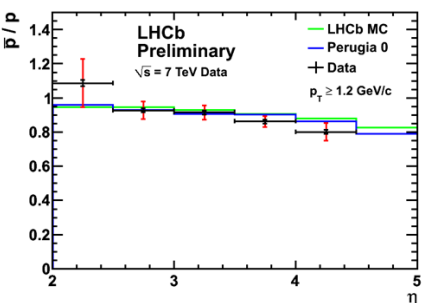

(f)

Figure 3: The production ratio $\bar{p} / p$ from $p p$ collisions at $\sqrt{s}=0.9 \mathrm{TeV}$ and $7 \mathrm{TeV}$ as a function of pseudorapidity, $\eta$, compared to the predictions of PYTHIA tunings Perugia 0 and LHCb MC in bins of transverse momentum, $p_{T}$. The vertical error bars give the total statistical and systematic uncertainties. 


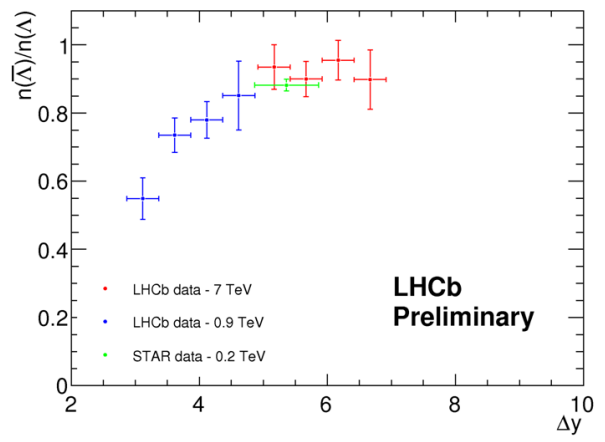

(a) $\bar{\Lambda} / \Lambda$

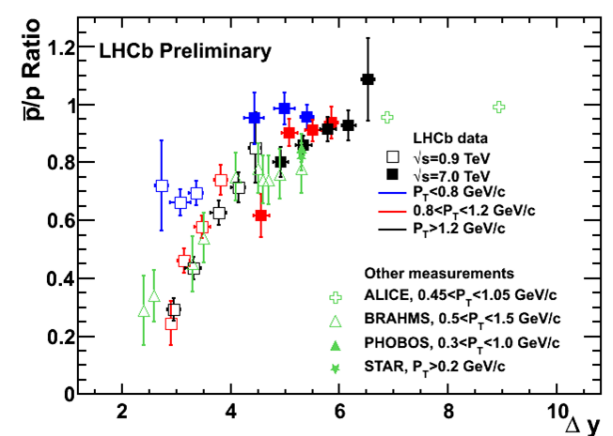

(b) $\bar{p} / p$

Figure 4: The production ratios $\bar{\Lambda} / \Lambda$ and $\bar{p} / p$ at LHCb compared as a function of rapidity loss, $\Delta y$, show good agreement between results at $\sqrt{s}=0.9 \mathrm{TeV}$ and $7 \mathrm{TeV}$ as well as with past experimental results.

\section{Comparison with past experiments}

The $\bar{\Lambda} / \Lambda$ and $\bar{p} / p$ results can be reformulated in terms of rapidity loss, $\Delta y=y_{\text {Beam }}-y_{\Lambda, p}$, where the beam rapidity is 6.9 at $\sqrt{s}=0.9 \mathrm{TeV}$ and 8.9 at $7 \mathrm{TeV}$. This change of variables effectively scales the results with beam energy allowing direct comparison between LHCb results at both collision energies and with results of past experiments (figure 5). Both ratios show good agreement with published experimental results.

\section{Conclusions}

The $K_{S}^{0}$ production cross section results are in good agreement with phenomenological models at the new collision environment of the LHC. Measurements of baryon suppression in strange quark hadronisation (from $\bar{\Lambda} / K_{S}^{0}$ ) suggest a significant overestimate, particularly from Perugia 0 . These results combined suggest an over all underestimate of strange particle production, either due to the strange quark production fraction or the total particle production.

Preliminary results on baryon number transport show good agreement with PYTHIA tunings for both $\bar{\Lambda} / \Lambda$ and $\bar{p} / p$ at $\sqrt{s}=7 \mathrm{TeV}$ but there is a hint that these ratios are overestimated at low energy and, for $\bar{p} / p$, this difference is shown to grow at lower $p_{T}$. Both ratios also show good agreement with published experimental results when shown as a function of rapidity loss, $\Delta y$.

\section{References}

[1] T. Sjöstrand, S. Mrenna and P. Skands, JHEP 05 (2006) 026.

[2] The LHCb collaboration, Physics Letters B 693 (2010) 69.

[3] M. Ferro-Luzzi, these proceedings.

[4] S. Borghi, these proceedings.

[5] P.Z. Skands, CERN-PH-TH-2010-113.

[6] The LHCb collaboration, CERN-LHCb-CONF-2010-011.

[7] The LHCb collaboration, CERN-LHCb-CONF-2010-009.

[8] A. Powell, these proceedings. 